\title{
Handheld diffuse optical breast scanner probe for cross-sectional imaging of breast tissue*
}

\author{
Majid Shokoufi ${ }^{\dagger}$ and Farid Golnaraghi \\ School of Mechatronic Systems Engineering \\ Simon Fraser University, 250-13450 102nd Avenue \\ Surrey, Canada, BC V3T $0 A 3$ \\ †mshokouf@sfu.ca
}

Received 25 July 2018

Accepted 5 February 2019

Published 14 March 2019

\begin{abstract}
Diffuse optical spectroscopy is a relatively new, noninvasive and nonionizing technique for breast cancer diagnosis. In the present study, we have introduced a novel handheld diffuse optical breast scan (DOB-Scan) probe to measure optical properties of the breast in vivo and create functional and compositional images of the tissue. In addition, the probe gives more information about breast tissue's constituents, which helps distinguish a healthy and cancerous tissue. Two symmetrical light sources, each including four different wavelengths, are used to illuminate the breast tissue. A high-resolution linear array detector measures the intensity of the back-scattered photons at different radial destinations from the illumination sources on the surface of the breast tissue, and a unique image reconstruction algorithm is used to create four cross-sectional images for four different wavelengths. Different from fiber optic-based illumination techniques, the proposed method in this paper integrates multi-wavelength light-emitting diodes to act as pencil beam sources into a scattering medium like breast tissue. This unique design and its compact structure reduce the complexity, size and cost of a potential probe. Although the introduced technique miniaturizes the probe, this study points to the reliability of this technique in the phantom study and clinical breast imaging. We have received ethical approval to test the DOB-Scan probe on patients and we are currently testing the DOB-Scan probe on subjects who are diagnosed with breast cancer.
\end{abstract}

Keywords: Breast cancer; diffuse optical spectroscopy; image reconstruction techniques; medical and biological imaging; optical breast phantom.

\section{Introduction}

According to the Canadian and American cancer societies, breast cancer is the most common cancer among women and is ranked as the second leading cause of cancer death in women in North America. ${ }^{1,2}$

\footnotetext{
${ }^{*}$ The ideas discussed herein have been filed for a US Patent titled: HANDHELD PROBE AND SYSTEM FOR IMAGING HUMAN TISSUE, Application No. CA/2018/050052, January 17, 2018.
}

This is an Open Access article published by World Scientific Publishing Company. It is distributed under the terms of the Creative Commons Attribution 4.0 (CC-BY) License. Further distribution of this work is permitted, provided the original work is properly cited. 
Ultrasound, X-ray mammography and magnetic resonance imaging (MRI) are commonly used for breast cancer detection, where X-ray mammography is the primary screening technique. ${ }^{3}$ Although $\mathrm{X}$-ray mammography is the principle modality for breast cancer screening, it is recommended for women over the age of $50,{ }^{4}$ because it is difficult to distinguish the lesion from the image for younger women whose breasts are much denser. In addition, it has a potential health risk due to the effect of ionizing radiations. ${ }^{5,6}$ Hereby, due to X-ray mammography's limits, researchers have been encouraged to use different breast cancer detection modalities, such as diffuse optical tomography, impedance spectroscopy or elastography. ${ }^{7}$

Over the past three decades, there have been significant advancements in optoelectronics components and fiber optics. In parallel with these developments, near-infrared (NIR) optical imaging, in association with optoelectronic devices and fiber optics, have also been developed rapidly. NIR optical imaging approaches, known as a noninvasive and promising method for compositional and functional imaging of extremely scattering media, have been advanced for breast cancer diagnosis..$^{7,8}$ The optical properties of the breast tissue have been widely studied and published in Refs. 9-12. According to these studies, among the four main constituents of the breast tissue, fat, water $\left(\mathrm{H}_{2} \mathrm{O}\right)$, deoxy-hemoglobin $(\mathrm{Hb})$ and oxy-hemoglobin $\left(\mathrm{HbO}_{2}\right)$, the absorption offat and waterare low in the wavelength spectrum from $650 \mathrm{~nm}$ to $1100 \mathrm{~nm}$, which allows light to travel deeply into the breast tissue. ${ }^{9,13-15}$

A number of handheld diffuse optical probes have been implemented and tested for breast cancer detection, as reported in Refs. 16-25. Almost all of the probes are designed in reflectance geometry to measure optical properties of the breast tissue. ${ }^{16,24}$ Laser light sources or fiber coupled laser light sources in NIR range are used to determine the concentration of main chromophores in the breast tissue related to sensing anomalies. No handheld functional and compositional breast cross-sectional imaging devices have been reported to date.

In the present study, a novel handheld diffuse optical breast scanning probe was designed and developed for functional and compositional cross-sectional imaging of the breast tissue. To evaluate performance and usability of the device we tested the probe on breast tissue-mimicking phantoms, which have similar optical properties as breast tissue, before using the device on human subjects. Here, the prototype of the DOB-Scan probe is introduced utilizing multi-wavelength encapsulated light emitting diodes (eLED), which contain four NIR wavelengths, $690 \mathrm{~nm}, 750 \mathrm{~nm}, 800 \mathrm{~nm}$ and $850 \mathrm{~nm}$, as illumination sources. The LEDs are set $0.5 \mathrm{~mm}$ apart from each other and perform as multiwavelengths pointed illumination source in such a scattering medium. In our previous study, ${ }^{23}$ we experienced that using eLED as an illumination source reduces complexity, size and cost of the probe. In this study, we utilized a linear array detector with 2048 sensors, to measure the intensity of the backscattered photons on the surface of the breast to create functional and compositional cross-sectional images of the breast tissue. The illumination source wavelengths are chosen because breast fat and water has the low absorption in mentioned wavelengths. ${ }^{23}$ Solid results from phantom study motivated us to conduct the first phase of clinical study at Jim Pattison Outpatient Care and Surgery Centre (JPOCSC) on subjects who have been diagnosed with breast cancer.

\section{Instrument Design}

Diffused optical imaging takes advantage of diffraction differences in the NIR spectrum of water, fat and blood. The hand-held DOB-Scan probe use novel technique for breast imaging, which consists of a linear array detector, two multiwavelengthlight-emitting diodes, custom hardware interface and software with unique image reconstruction algorithm. Figures 1(a) and 2(b) present a sketch and photograph of the DOB-Scan probe, respectively.

Eight NIR light sources are used to illuminate the breast tissue through two eLEDs. A Schematic diagram of the instrument's internal hardware design is shown in Fig. 2.

Each wavelength illuminates the under-test tissue sequentially, and the linear CCD array collects back-scattered light on the skin and converts it to an analog voltage. Analog signals are then converted to digital signals by an analog to digital converter (ADC) and passed to the main processor. The microcontroller unit (MCU) is responsible for controlling the eLEDs, ADC and CCD, collecting digital data from the ADC and transferring data to host computer for further analysis. 


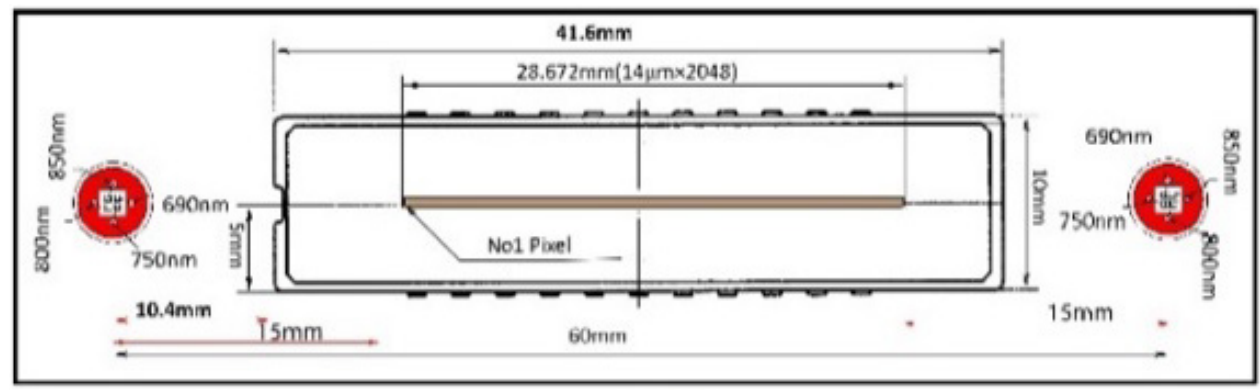

Linear array $C C D$

Multi-wavelength light source

(a)

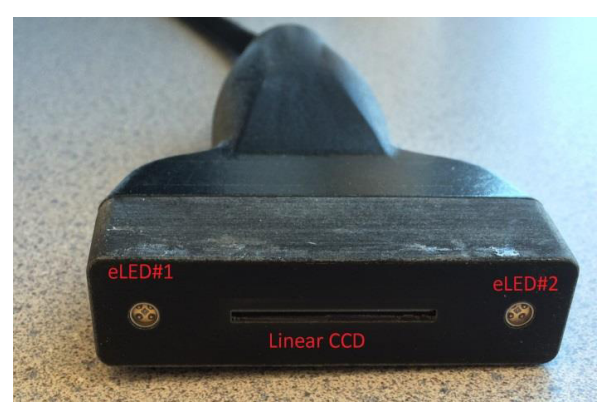

(b)

Fig. 1. (a) Front-end light sources and linear charge coupled device (CCD) orientation on the probe and (b) Photograph of DOB-Scan probe.

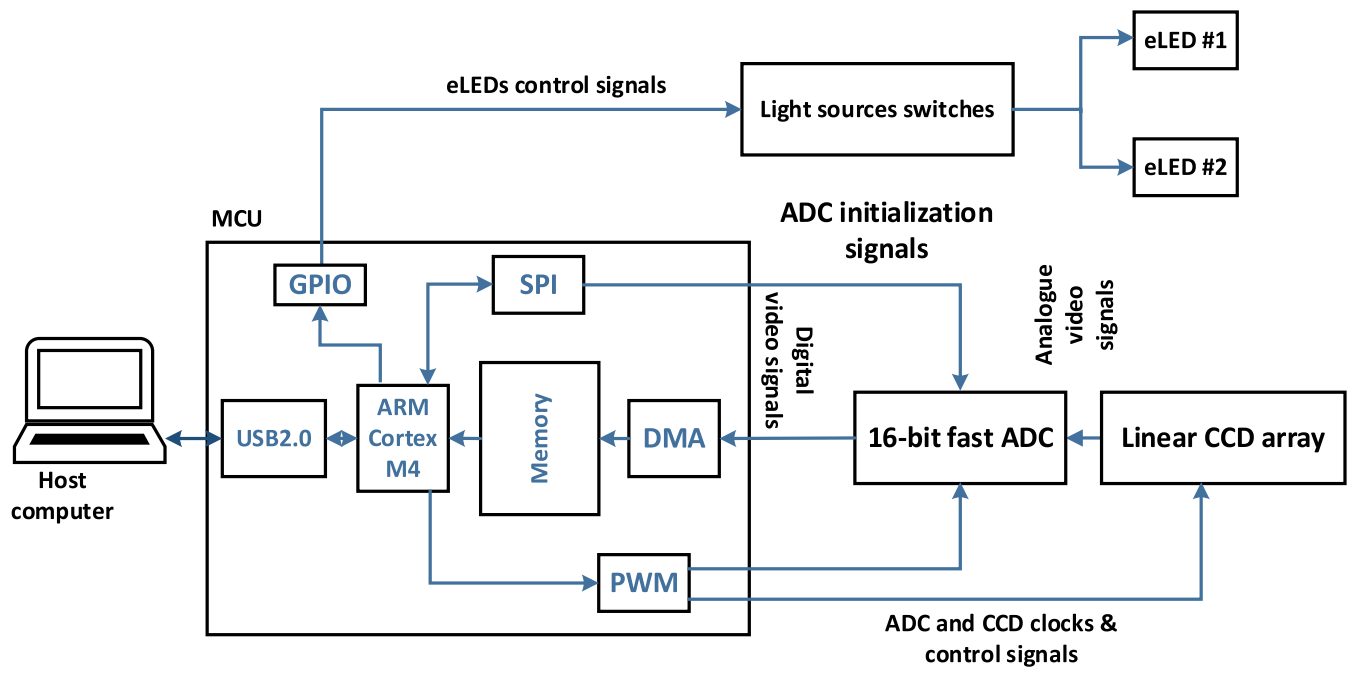

Fig. 2. Schematic of the cross-sectional diffuse optical imaging instrument.

\subsection{Light illumination}

Two eLEDs (Marubeni America Corporation L690/ $750 / 800 / 850$ ) are used in the probe, located $15 \mathrm{~mm}$ away from each end-side of the detector as shown in Fig. 1. Each eLED includes four NIR wavelengths:
$690 \mathrm{~nm}, 750 \mathrm{~nm}, 800 \mathrm{~nm}$ and $850 \mathrm{~nm}$. Penetrations of photons in these wavelengths are maximized due to low absorption of breast fat and water at the chosen wavelengths. The light sources can turn on and off individually or sequentially with a period of 
$83 \mathrm{~ms}$, which is sufficient for functional imaging of biological tissue. The intensity of each LED can be calibrated through the software.

\subsection{Light detection}

The light detector plays an important role in the instrument. In order to produce high-resolution images, it must have a large number of light sensors in small sizes and patches. The light sources are located at two ends of the detector and the backscattered photons are traveling in different paths to reach the photodetectors. The pixels in the photodetector located closer to the active light source will be exposed to higher light intensity, and the ones that are placed far from the active light source will receive lower light intensity. Therefore, the photodetectors must have a large dynamic range to allow measurement for both small and large sourcephotodetector separations. The light detector (ILX511, SONY) used for the proposed probe is a linear array CCD, which has total 2086 pixels in one frame. 32 pixels at the beginning of the frame and 6 pixels at the end of the frame are dummy pixels. Therefore, the photodetector has 2048 active pixels with $14 \mu \mathrm{m}$ pixel pitch; consequently, the effective imaging area is $28.672 \mathrm{~mm}(2048 \times 14 \mu \mathrm{m})$. A circuit is specially designed to maximize the functionality of the CCD array.

\subsection{Signal digitization and processing}

A MATLAB@ (MATLAB R2012b, MathWoks ${ }^{\circledR}$ ) based graphical user interface (GUI) gives the user complete control over the hardware via USB2.0, allowing them to collect, store and analyze data on the host computer. The GUI is comprised of a series of tabs and collected data graphs for various operations including manual and periodic light source selection, CCD integration time setting and data collection. Upon launching the software interface, the user must manually select illumination wavelengths, or set it to periodic rotation in LEDs control panel. In the manual-mode, 24 frames per second are collected from the image sensor, which is sufficient for functional imaging of biological tissue. In the periodic-mode, each wavelength is illuminated for $200 \mathrm{~ms}$ and the data is collected with the same rate. In this mode, eight sets of data are collected while different wavelengths illuminate sequentially into the biological tissue, and in order to measure the ambient light effect on the image sensor, one more frame is collected while all the LEDs are turned off.

\section{Evaluation and Image Reconstruction Algorithm}

\subsection{Theory and background}

In this section, we briefly overviewed NIR photons migration in biological tissue and the optical properties of the breast tissue. The optical properties of breast tissues including $\mathrm{Hb}, \mathrm{HbO}_{2}$, water and fat are widely studied and published in Refs. 9-12. These studies demonstrate that the absorption of fat and water are low in the NIR range, which allows photons to penetrate deeply into the breast tissue..$^{9,13-15}$ In a highly scattering medium such as breast tissue, photon traveling paths can be simulated by diffusion equation given in (1)..$^{9,13-15,23}$

$$
\nabla D(d) \nabla \phi_{d}(d)-\mu_{a} C_{m} \phi_{d}(d)=-S(d),
$$

where $\phi_{d}$ represents the intensity at the detector located $d \mathrm{~mm}$ away from the illumination source $S(d) . C_{m}$ and $\mu_{a}$ are the photons velocity in the medium and absorption coefficient, respectively. $D$ represents diffusion coefficient. ${ }^{14,23}$ The inverse solution for diffusion equation, given in (2), for a semiinfinite homogeneous medium is used to extract optical properties of the medium for different light paths when the illumination sources and detector are oriented in reflectance geometry. ${ }^{14,16,23,26}$

$$
\begin{aligned}
R(d)= & \frac{1}{4 \pi \mu_{t}^{\prime}}\left[\left(\mu_{\mathrm{eff}}+\frac{1}{r_{1}}\right) \frac{\exp \left(-\mu_{\mathrm{eff}} r_{1}\right)}{r_{1}^{2}}\right. \\
& \left.+(1.33 A+1)\left(\mu_{\mathrm{eff}}+\frac{1}{r_{2}}\right) \frac{\exp \left(-\mu_{\mathrm{eff}} r_{2}\right)}{r_{2}^{2}}\right], \\
r_{1}= & \left(\left(\frac{1}{\mu_{t}^{\prime}}\right)^{2}+d^{2}\right)^{1 / 2}, \\
r_{2}= & \left(\left(\frac{\left(\frac{4}{3}\right) A+1}{\mu_{t}^{\prime}}\right)^{2}+d^{2}\right)^{1 / 2}, \\
\mu_{\mathrm{eff}}= & \left(3 \mu_{a} \mu_{t}^{\prime}\right)^{1 / 2}, \\
\mu_{t}^{\prime}= & \mu_{a}+\mu_{s}^{\prime}=\mu_{a}+(1-g) \mu_{s},
\end{aligned}
$$

where the parameters are defined as follows ${ }^{14,23}$ :

$\mu_{s} \quad$ Scattering coefficient

$\mu_{s}^{\prime} \quad$ Reduced scattering coefficient

$\mu_{\text {eff }}$ Effective attenuation coefficient 
$\mu_{a} \quad$ Absorption coefficient

$\mu_{t}^{\prime} \quad$ Total attenuation coefficient

$R(d)$ Measured scattered photon intensity at the distance of $d$ from the illumination source

\section{$A \quad$ Internal reflection parameter}

The diffusion equation is effective for large sourcedetector separation, $r>10 \times\left(\mu_{a}+\mu_{s}\right)^{-1}$ and in the case when the absorption coefficient is much smaller than the scattering coefficient, $\mu_{a} \ll \mu_{s}^{\prime} .{ }^{11,23}$

\subsection{Evaluation method}

According to Eq. (2), since absorption coefficient $\left(\mu_{a}\right)$ of the breast tissue is much smaller than reduced scattering coefficient $\left(\mu_{s}^{\prime}\right)$, fluctuation in $\mu_{a}$ will not have a remarkable variation on total attenuation coefficient $\left(\mu_{t}^{\prime}\right)$. Several studies have also demonstrated that there are no remarkable changes on the $\mu_{t}^{\prime}$ between normal and cancerous breast tissue. ${ }^{23,27}$ These studies conclude that $\mu_{a}$ and $\mu_{s}^{\prime}$ of the breast tissue are in the range of $0.002 \mathrm{~mm}^{-1}$ to
$0.012 \mathrm{~mm}^{-1}$ and $0.6 \mathrm{~mm}^{-1}$ to $1.5 \mathrm{~mm}^{-1}$, respectively, in the NIR spectrum range. ${ }^{9,13,23,28}$ In our previous study, we have also shown that the variation on $\mu_{t}^{\prime}$ does not have notable results on the backscattered photons at the photodetector position. ${ }^{23}$ Therefore, it can be assumed that $\mu_{t}^{\prime}$ remains the same in breast tissue.

Equation (2) overall contains two unknowns if elaborated, $\mu_{s}^{\prime}$ and $\mu_{a}$. The remaining parameters can be expressed using these two variables. Since there are large number of data points from CCD, any two of them with different distances can solve for $\mu_{s}^{\prime}$ and $\mu_{a}$, which quantify the effect of scattering and absorption in breast tissue separately.

As mentioned in previous sections, absorption at the utilized wavelengths in the DOB-Scan probe occurs in breast tissue due to four main absorbers. We can further use four different wavelengths in the DOB-Scan probe to extract the concentrations of the four absorbers in different locations of breast tissue underneath the probe with the following direct approach method:

$$
\left[\begin{array}{c}
\mu_{a}(690 \mathrm{~nm}) \\
\mu_{a}(750 \mathrm{~nm}) \\
\mu_{a}(800 \mathrm{~nm}) \\
\mu_{a}(850 \mathrm{~nm})
\end{array}\right]=\left[\begin{array}{cccc}
\varepsilon_{\mathrm{Hb}}(690 \mathrm{~nm}) & \varepsilon_{\mathrm{HbO}_{2}}(690 \mathrm{~nm}) & \varepsilon_{\mathrm{H}_{2} \mathrm{O}}(690 \mathrm{~nm}) & \varepsilon_{\mathrm{Fat}}(690 \mathrm{~nm}) \\
\varepsilon_{\mathrm{Hb}}(750 \mathrm{~nm}) & \varepsilon_{\mathrm{HbO}_{2}}(750 \mathrm{~nm}) & \varepsilon_{\mathrm{H}_{2} \mathrm{O}}(750 \mathrm{~nm}) & \varepsilon_{\mathrm{Fat}}(750 \mathrm{~nm}) \\
\varepsilon_{\mathrm{Hb}}(800 \mathrm{~nm}) & \varepsilon_{\mathrm{HbO}_{2}}(800 \mathrm{~nm}) & \varepsilon_{\mathrm{H}_{2} \mathrm{O}}(800 \mathrm{~nm}) & \varepsilon_{\mathrm{Fat}}(800 \mathrm{~nm}) \\
\varepsilon_{\mathrm{Hb}}(850 \mathrm{~nm}) & \varepsilon_{\mathrm{HbO}_{2}}(850 \mathrm{~nm}) & \varepsilon_{\mathrm{H}_{2} \mathrm{O}}(850 \mathrm{~nm}) & \varepsilon_{\mathrm{Fat}}(850 \mathrm{~nm})
\end{array}\right] \times\left[\begin{array}{c}
C_{\mathrm{Hb}} \\
C_{\mathrm{HbO}_{2}} \\
C_{\mathrm{H}_{2} \mathrm{O}} \\
C_{\mathrm{Fat}}
\end{array}\right]
$$

where $C$ and $\varepsilon$ are the total concentration of the chromophore, and the molar absorption extinction coefficient in the path length of the scattered light in the tissue. The concentration distribution of four absorbers can be calculated by solving Eq. (3) for different light paths to reconstruct images for the absorbers and visualizing their variation in the breast tissue.

\subsection{Image resolution algorithm}

The raw data,obtained from 2048 pixels in CCD array for 2 light sources and 4 wavelengths, are collected from the surface of the tissue. Analytical method is used for converting the surface-measured light intensity into two-dimensional cross-sectional images of the chromophores. While the tissue is illuminated by each wavelength sequentially, the absorption coefficient of each light path is calculated by utilizing Eq. (2). In order to reduce the effect of sensors' noise on the measurements, we reduced the number of measurement points to 128 $\left(P_{1}\right.$ to $\left.P_{128}\right)$ by averaging data every 16 pixels. We assume that the origin of the coordinate plane is located in the center of light source \#1 as showed in Fig. 3.

Two sets of data are collected for each wavelength: (1) while eLED \#1 is on and (2) while eLED \#2 is on. Assume we want to calculate the total absorption coefficient at location $\left(X_{1}, Y_{1}\right)$ as shown in Fig. 3. The illuminated light from eLED \#1 travels along the path $m$ and captured by the CCD, and then the intensity of scattered light is measured by the pixel $P_{m}$. A similar process occurs while eLED \#2 is on. The illuminated light from eLED \#2 travels along the path $n$ and scattered light is measured by the pixel $P_{n}$. Finally, the absorption coefficient at $\left(X_{1}, Y_{1}\right)$ is calculated as a superposition of two light path lengths of illuminated light from eLED \#1 and eLED \#2.

At this stage of the study, we only extract the absorption information using Eq. (2). The equation 


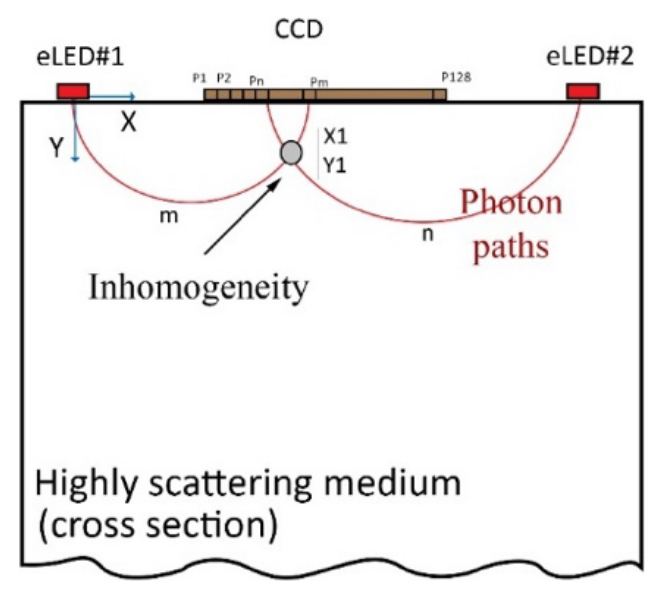

Fig. 3. Sketch of light propagation in highly scattering tissue.

contains two unknowns, reduced scattering coefficient $\left(\mu_{s}^{\prime}\right)$ and absorption coefficient $\left(\mu_{a}\right)$. Since there are the same wavelength at both side of CCD array, two equations can be established and used for solving two unknowns. A large value of the absorption coefficient indicates that there exists a higher concentration of chromophores comparing to other areas, which shown as orange or red color in the image. The constituent of the mass can be roughly distinguished by the wavelength, for example, if the images show a region with higher absorption in $800 \mathrm{~nm}$ or $850 \mathrm{~nm}$, it is highly possible that there is a water-filled lesion at that location, because water has the relative higher absorption among all four constituents in these two wavelengths. We are continuing the study to extract the composition of the four constituents in breast tissue, as demonstrated in Eq. (3), and improving the algorithm to provide more accurate and detail information.

Table 1 presents a brief specification of the DOB-Scan probe described above.

Table 1. DOB-Scan Probe characteristics and parameter.

\begin{tabular}{lc}
\hline Parameter & Value \\
\hline Wavelength & $2 \times(690 \mathrm{~nm}, 750 \mathrm{~nm}, 800 \mathrm{~nm}, 850 \mathrm{~nm})$ \\
Detector type & Linear CCD \\
Detector resolution & 2048 pixels \\
Image resolution & $128 \times 128$ pixels \\
Image length & $28.67 \mathrm{~mm}$ \\
Image depth & $24 \mathrm{~mm}$ \\
Detector sensitivity & $1800(\mathrm{~V} / \mathrm{Lx} . \mathrm{S}) @ 660 \mathrm{~nm}$ \\
Illumination tech. & Continuous wave \\
Max. frame rate & 24 \\
Power consumption & $100 \mathrm{~mA} @ 5 \mathrm{~V}$ \\
\hline
\end{tabular}

\section{Experimental Results}

\subsection{Phantom study}

To verify performance of the DOB-Scan probe, a study based upon tissue equivalent material was conducted. In this study, we used an incomplete (truncated) cylindrical shaped $(8 \mathrm{~cm}$ diameter and $28 \mathrm{~cm}$ length) Acetal resin $\operatorname{rod}\left(\right.$ Delrin $\left.{ }^{\circledR}\right)$ with similar optical properties to breast fat $\left(\mu_{s}^{\prime}=\right.$ $2.3 \mathrm{~mm}^{-1}$ and $\left.\mu_{a}=0.002 \mathrm{~mm}^{-1}\right)$ and Intralipid ${ }^{\circledR}$ to mimic background breast. ${ }^{23,29}$ Optical properties of these two materials are also well-documented and used in several studies. ${ }^{23,29-33}$ In order to mimic cancerous lesions, a $5 \mathrm{~mm}$ in diameter hole is drilled on the side wall of the cylinder; located $15 \mathrm{~mm}$ below the incomplete side of the Acetal resin rod, as shown Fig. 4. A solution of Intralipid ${ }^{\circledR} 20 \%$ emulsion (Fresenius Kabi Inc.), water and black ink (Higgins India Ink) is used as tumor-like liquid phantom and injected into the hole. ${ }^{16}$ Black India ink is used in this study because of its flat absorption in the NIR spectral range and its water-solubility properties.

In the first experiment, we used phantom \#1 with the hole filled with a tumor-like liquid phantom. The liquid-phantom is a solution consisting of $1 \mathrm{~L}$ water, $50 \mathrm{~mL}$ Intralipid $^{\circledR}$ and $1.5 \mathrm{~mL}$ India black ink. In this case, we put the probe in three different locations on the phantom, where the center line of the probe (middle of the CCD) is aligned: (a) with the center of the hole, (b) $7 \mathrm{~mm}$ left to the hole, (c) $7 \mathrm{~mm}$ right to the hole as shown in Fig. 5 .

Figure 6 shows the reconstructed cross-sectional images of the phantom for the wavelength of $690 \mathrm{~nm}$ while the probe is aligned: (a) with the center of the hole, (b) $7 \mathrm{~mm}$ left to the hole, (c) $7 \mathrm{~mm}$ right to the hole. The red colors on the images show higher absorption, which indicates the location of the hole on the phantom. The $X$ and $Y$ axes on the images

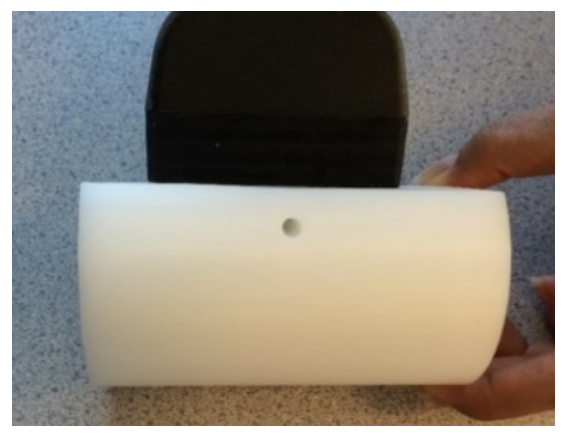

Fig. 4. The breast phantom constructed from Acetal resin rod. 


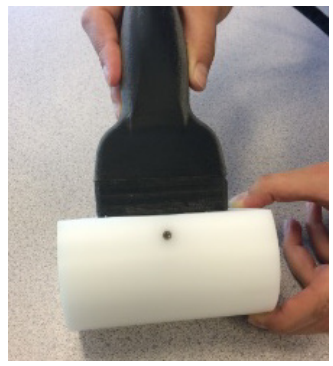

(a)

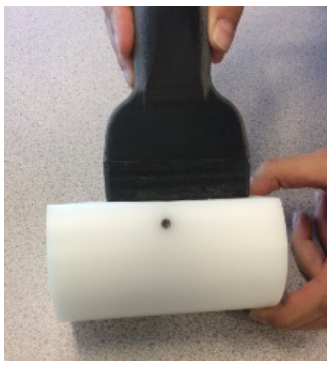

(b)

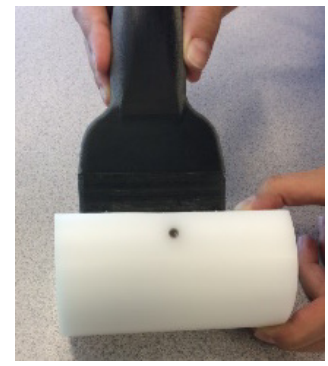

(c)

Fig. 5. DOB-Scan probe in three different locations on the phantom. The center line of the probe is aligned: (a) with the center of the hole, (b) $7 \mathrm{~mm}$ left to the hole, (c) $7 \mathrm{~mm}$ right to the hole.

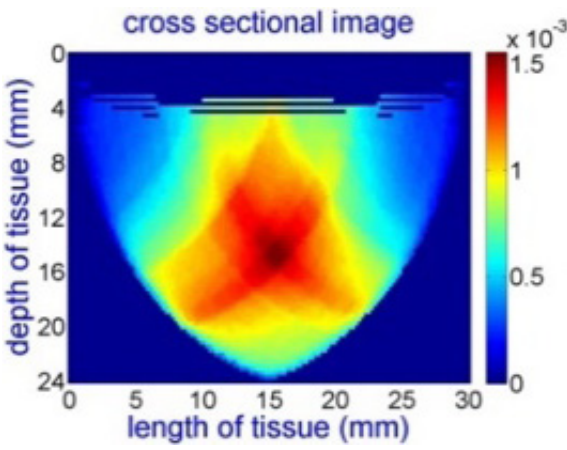

(a)

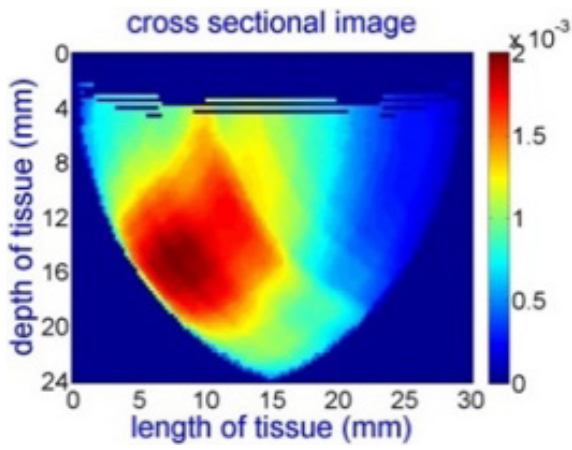

(b)

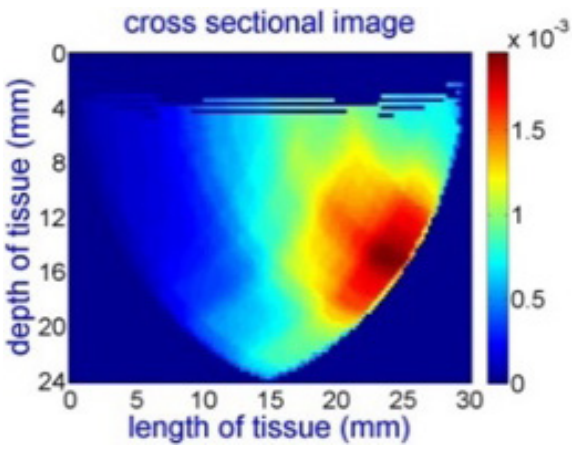

(c)

Fig. 6. Reconstructed cross-sectional images of the phantom for the wavelength of $690 \mathrm{~nm}$ while the center line of the probe is aligned: (a) with the center of the hole, (b) $7 \mathrm{~mm}$ left to the hole, (c) $7 \mathrm{~mm}$ right to the hole.

represent the length and depth of the cross-sectional imaging area. The vertical color bars next to the images show the absorption coefficient of different colors on the images (unit: $\mathrm{mm}^{-1}$ ). Since black India ink has uniform absorption in the NIR spectrum, we have similar images for all wavelengths $(690 \mathrm{~nm}, 750 \mathrm{~nm}, 800 \mathrm{~nm}$ and $850 \mathrm{~nm})$. The test results demonstrate that the probe can capture the inhomogeneity in the phantom.

In the second experiment, we evaluated the probe's ability to accurately distinguish different absorbers such as $\mathrm{Hb}$ and $\mathrm{HbO}_{2}$ in different wavelength. We used protein assay reagent (PAR) to form an inhomogeneity inside the phantom \#2, which has similar optical properties to $\mathrm{HbO}_{2}$ in the NIR spectrum range. A solution of $1.5 \mathrm{~mL}$ Pierce ${ }^{\mathrm{TM}}$ $660 \mathrm{~nm}$ PAR (Thermo Scientific ${ }^{\mathrm{TM}}$ Inc.) mixed with $10 \mu \mathrm{L}$ prediluted protein assay standards: Bovine Serum Albumin (BSA), with $1000 \mu \mathrm{g} / \mathrm{mL}$ in $0.9 \%$ saline and $0.05 \%$ sodium azide was used to make a PAR. The absorption coefficient of RBSA at $690 \mathrm{~nm}$ is $0.17 \mathrm{~mm}^{-1}$ which is much higher than its absorption coefficient at $750 \mathrm{~nm}, 800 \mathrm{~nm}$ and $850 \mathrm{~nm}\left(0.015 \mathrm{~mm}^{-1}\right){ }^{23}$

Figure 7 shows cross-sectional images for the wavelength of $690 \mathrm{~nm}$ while the probe is placed at different locations on the phantom. Since the absorption coefficient of the RBSA at this wavelength is much higher than the surrounding material, the probe can capture this difference. However, for the $750 \mathrm{~nm}, 800 \mathrm{~nm}$ and $850 \mathrm{~nm}$ wavelengths, the probe did not capture the inhomogeneity due to the similar absorption coefficient of RBSA with the surrounding material as illustrated in Fig. 8, showing the wavelength of $800 \mathrm{~nm}$ as an example.

In order to further evaluate the effectiveness of the probe in monitoring different concentration of chromophores, we conducted an additional experiment using the same shape of Acetal resin rod (phantom \#3). The tumor phantom was formed by adding India black in into a reference solution of water and intralipid ${ }^{\circledR}(50 \mathrm{~mL} / \mathrm{L})$. In this experiment, the amount of India black ink was increased from $0.125 \mathrm{~mL} / \mathrm{L}$ to $0.75 \mathrm{~mL} / \mathrm{L}$ with an increment of 


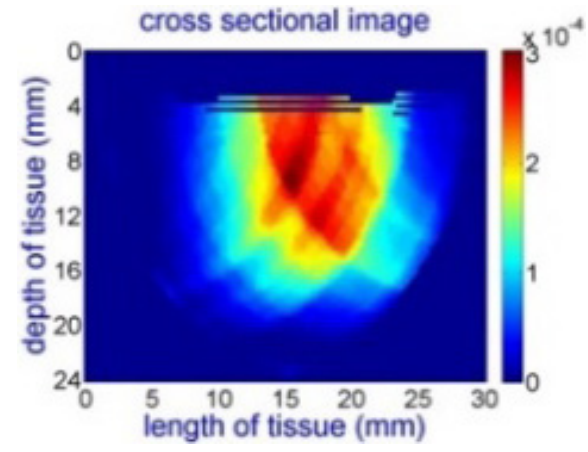

(a)

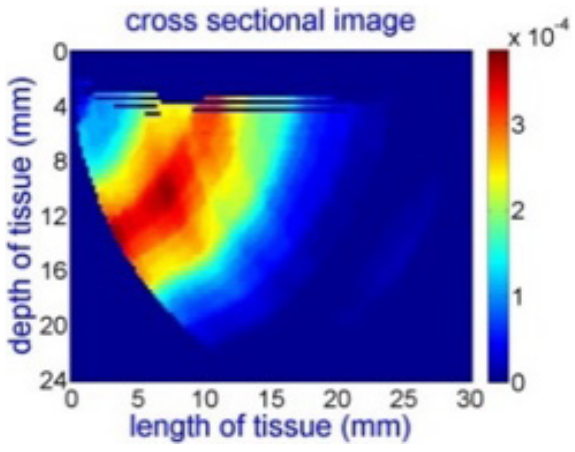

(b)

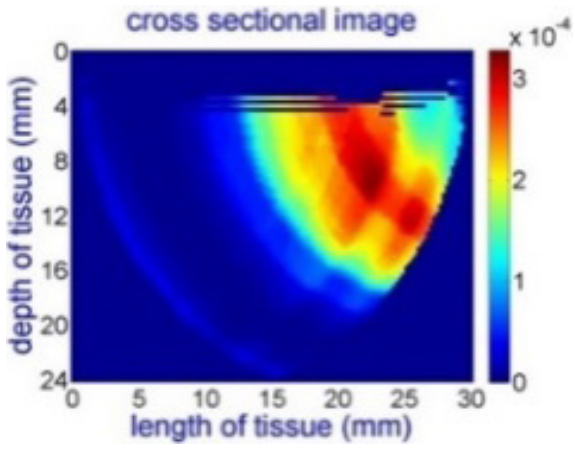

(c)

Fig. 7. Reconstructed cross-sectional image for the RBSA phantom for the wavelength of $690 \mathrm{~nm}$ while the center line of the probe is aligned: (a) with the center of the hole, (b) $7 \mathrm{~mm}$ left to the hole, (c) $7 \mathrm{~mm}$ right to the hole for $690 \mathrm{~nm}$ light source.

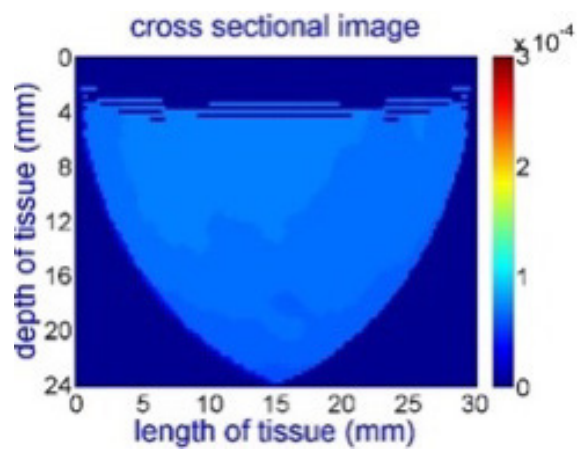

(a)

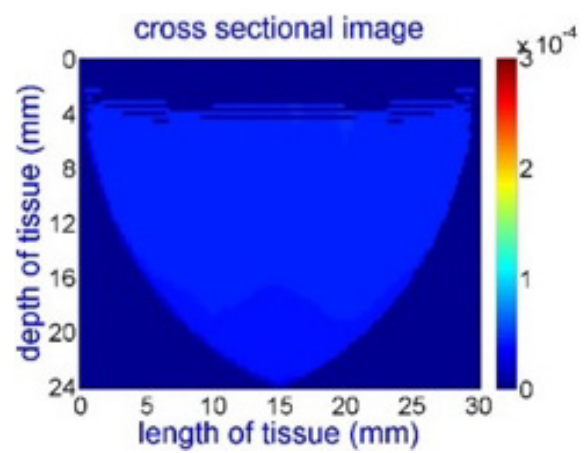

(b)

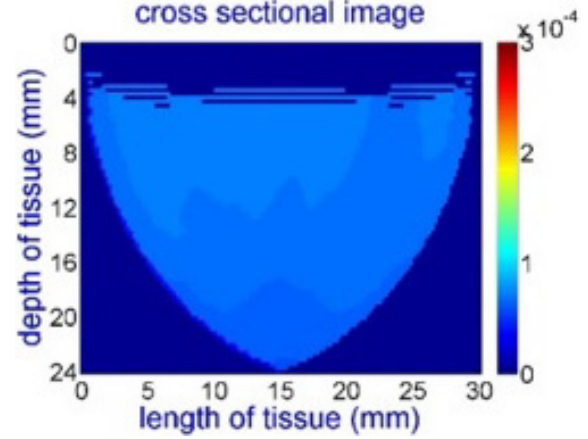

(c)

Fig. 8. Reconstructed cross-sectional images of the RBSA phantom for the wavelength of 800 nm while the center line of the probe is aligned: (a) with the center of the hole, (b) $7 \mathrm{~mm}$ left to the hole, (c) $7 \mathrm{~mm}$ right to the hole for $750 \mathrm{~nm}, 800 \mathrm{~nm}$ and $850 \mathrm{~nm}$ light sources.

$0.125 \mathrm{~mL} / \mathrm{L}$. The absorption coefficient of theinhomogeneity was gradually increasing butthe scattering coefficient of the medium was not affected by the change of ink concentration.

Four images for each step and 24 images in total were reconstructed. As mentioned previously,India black ink has a uniform absorption spectrum in the NIR range. Therefore, images for four wavelengths in the same step are identical. Figure 9 shows the images for $690 \mathrm{~nm}$ as an example.

Figures 9(a)-9(f) clearly, show that the area of red color increases as the concentration of chromophores increases. As a result, we can conclude that the DOBScan probe is capable of capturing changes in the absorption coefficient of the tumor phantom.

\subsection{Clinical study}

Promising results from the phantom study motivated us to extend data collection over the actual cancerous tissue to further evaluate the probe's performance. In this study, we tested the DOB-Scan probe to identify malignant breast tumors using contralateral healthy body tissueas a control. This research was conducted in collaboration with Fraser Health and performed under the Fraser Health Research Ethics Board and SFU Ethics Board (principals: Dr. Farid Golnaraghi, Simon Fraser University and Dr. Rhonda Janzon, Jim Pattison Outpatient Care and Surgery Centre - JPOCSC). The preliminary results for two out of 20 cases are presented next.

In the first case study, the subject's latest mammography confirmed that there was a mass located in the left breast $54 \mathrm{~mm}$ away from the nipple at the 10 o'clock position (size $1 \times 0.8 \times$ $0.7 \mathrm{~cm}$ ). The mammography image for the second case study confirmed that there was a mass lesion located approximately $2 \mathrm{~cm}$ in size with ill-defined margins at the 1 o'clock position in the right breast. 


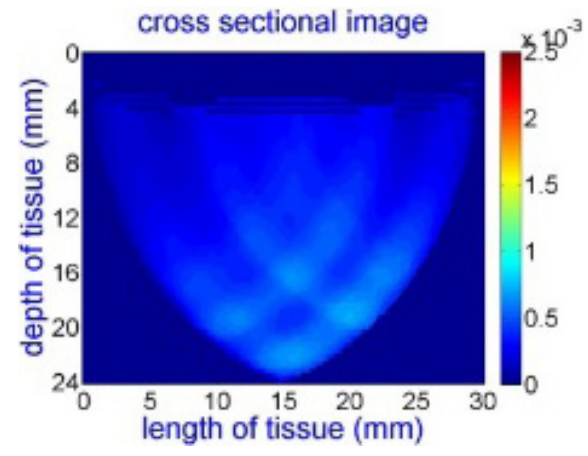

(a)

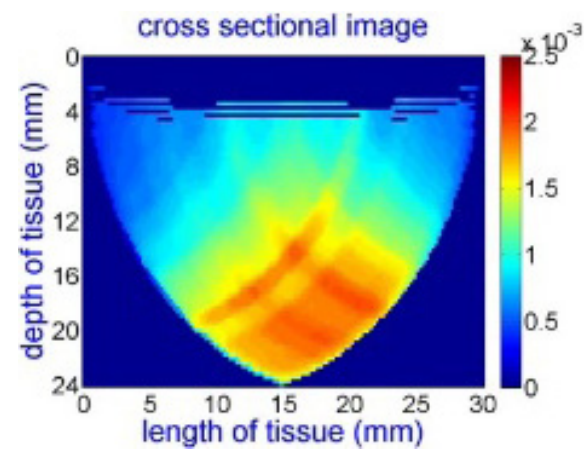

(d)

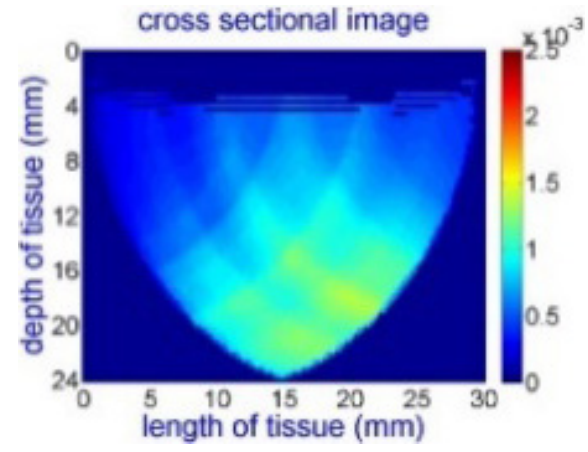

(b)

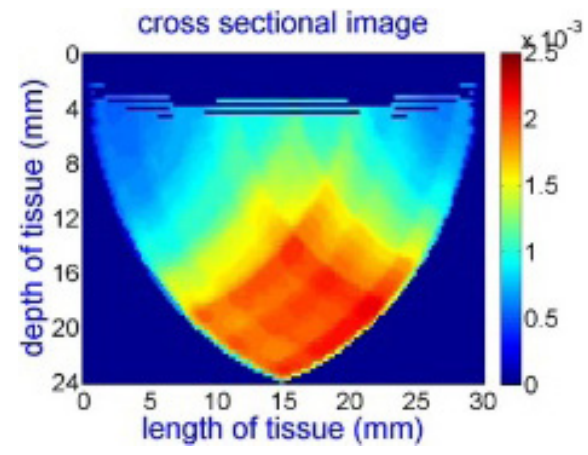

(e)

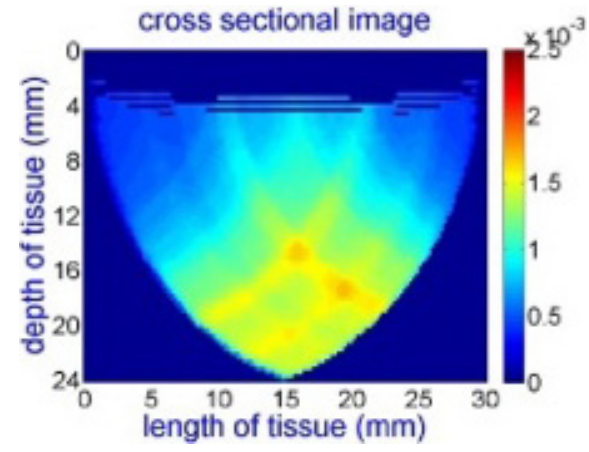

(c)

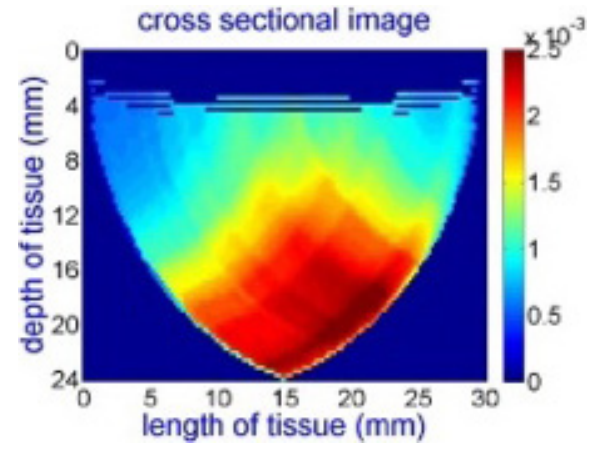

(f)

Fig. 9. Reconstructed images for the wavelength of $690 \mathrm{~nm}$ while concentration of India black ink is gradually increased. (a) $0.125 \mathrm{~mL} / \mathrm{L}$, (b) $0.25 \mathrm{~mL} / \mathrm{L}$, (c) $0.375 \mathrm{~mL} / \mathrm{L}$, (d) $0.5 \mathrm{~mL} / \mathrm{L}$, (e) $0.625 \mathrm{~mL} / \mathrm{L}$ and (f) $0.75 \mathrm{~mL} / \mathrm{L}$.

In both cases, the cancerous lesions were located at the lower outer quadrant of the breast.

The reconstructed images for both cases in four different wavelengths $(690 \mathrm{~nm}, 750 \mathrm{~nm}, 800 \mathrm{~nm}$, $850 \mathrm{~nm}$ ) are presented in Figs. 10 to 13 while the probe is positioned on the cancerous location and its contralateral spot on the healthy breast.

Case study \#1:

Figure 10 presents reconstructed images captured with the DOB-Scan probe on the cancerous lesion. The dark red color area on the images represents more vascular activity in comparison to healthy tissue caused by tumor cells.

Figure 11 shows captured images from the healthy breast (right breast) at the contralateral location of cancerous one, as there is no sign of a tumor in this breast. The area in red color on the top right side of the healthy breast images are caused by detector's saturation.

Case study \#2:

Figure 12 presents reconstructed images for the left (cancerous) breast. The images show that the

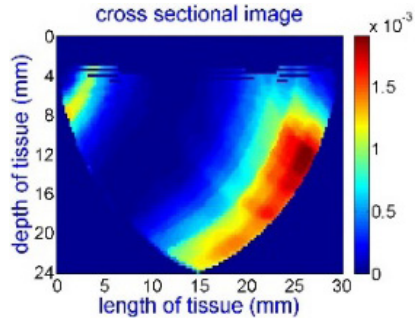

(c)

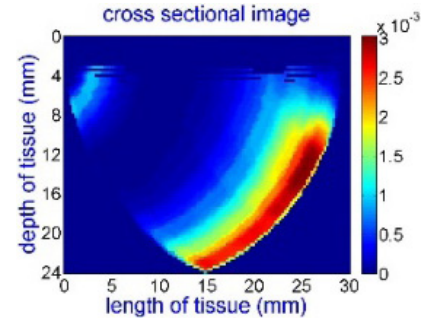

(d)

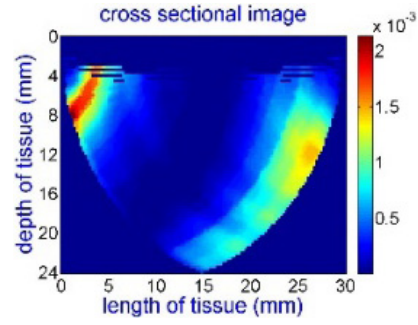

(b)

Fig. 10. Case study \#1: reconstructed images from collected data using the DOB-Scan probe in four wavelengths ((a) $690 \mathrm{~nm}$, (b) $750 \mathrm{~nm}$, (c) $800 \mathrm{~nm}$ and (d) $850 \mathrm{~nm}$ ), left breast, cancerous lesion located at 10 o'clock position and 54 mm away from the nipple. 


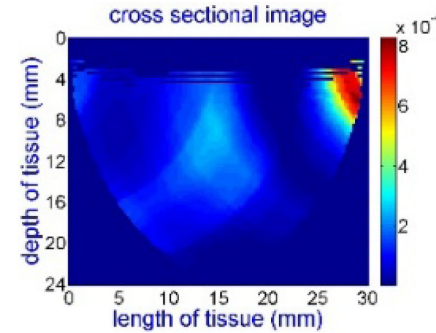

$690 \mathrm{~nm}$

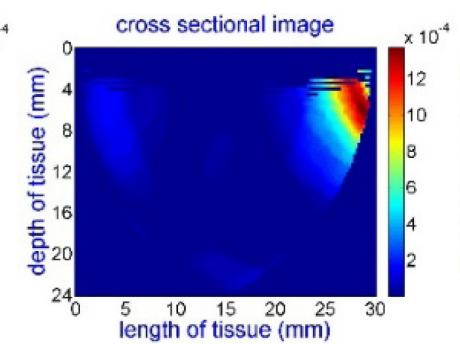

$750 \mathrm{~nm}$

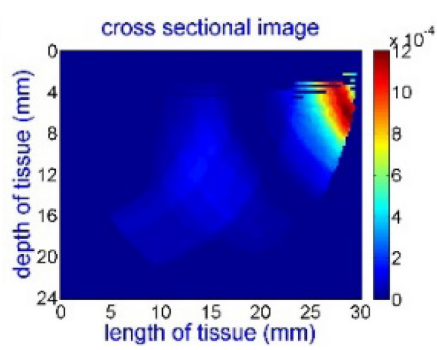

$800 \mathrm{~nm}$

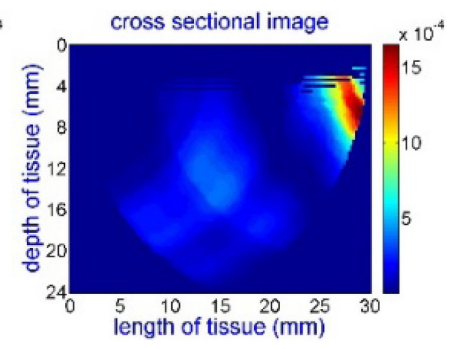

$850 \mathrm{~nm}$

Fig. 11. Case study \#1: reconstructed images from collected data using the DOB-Scan probe in four different wavelengths, right breast, healthy.

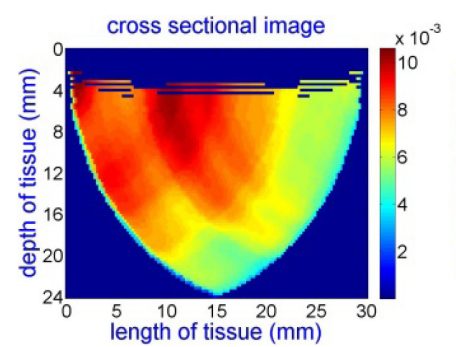

$690 \mathrm{~nm}$

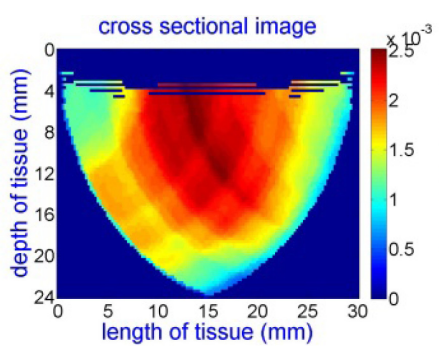

$750 \mathrm{~nm}$

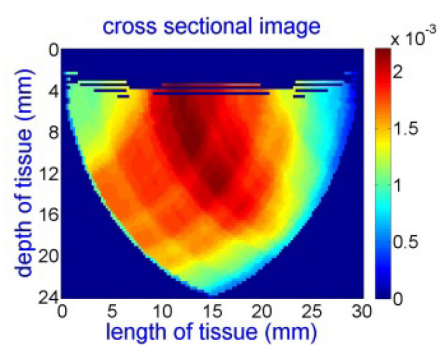

$800 \mathrm{~nm}$

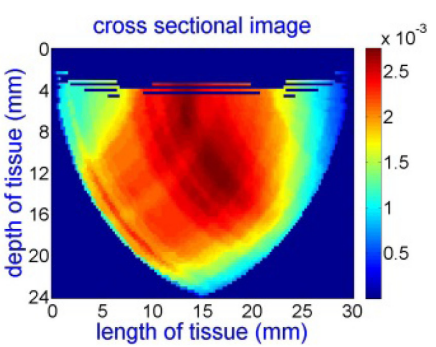

$850 \mathrm{~nm}$

Fig. 12. Case study \#2: reconstructed images from collected data using the DOB-Scan probe in four different wavelengths, right breast and cancerous lesion located $1.8 \mathrm{~cm}$ from nipple at 1 o'clock position.

probe captured the tumor correctly and it is in line with the mammography images provided by oncologist. Same as previous case, in order to have a comparison between healthy and cancerous lesion, the contralateral images of the healthy breast have been captured and presented in Fig. 13. The images for $800 \mathrm{~nm}$ and $850 \mathrm{~nm}$ show a lesion which has higher absorption coefficient in these two wavelength for the healthy breast. According to the absorption spectrum, ${ }^{34}$ we suspected there was a higher concentration of water at this spot comparing to the remaining area, as water has higher absorption coefficient at $800 \mathrm{~nm}$ and $850 \mathrm{~nm}$ than other constituents. After consulting with the patient's oncologists at JPOCSC, we were advised that there was a benign mass filled with water at that location. Therefore, as we expected the probe can also capture benign lesions and cysts as well.

The reconstructed preliminary images show that the DOB-San probe can distinguish a cancerous region from the surrounding healthy tissue. The cancerous region is observable in the images (red area) due to higher level of vascularization.

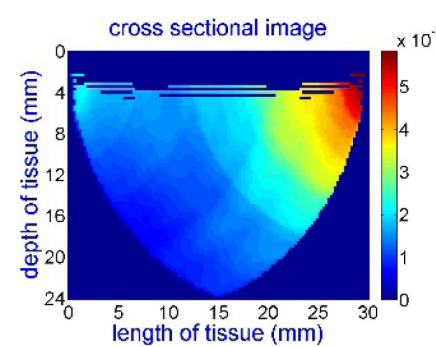

$690 \mathrm{~nm}$

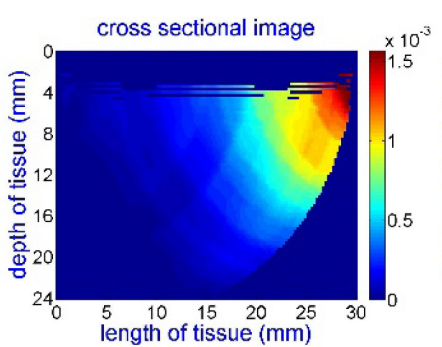

$750 \mathrm{~nm}$

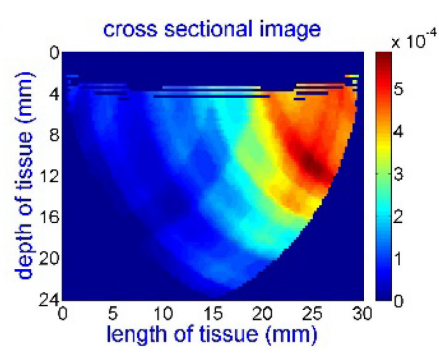

$800 \mathrm{~nm}$

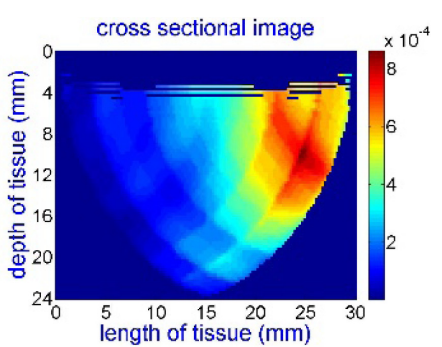

$850 \mathrm{~nm}$

Fig. 13. Case study \#2: reconstructed images from collected data using the DOB-Scan probe in four different wavelengths, left breast, healthy. 
The study enrollment is currently ongoing and will likely continue until mid 2019. Once we have a statistically viable number of case studies, these results will be presented in a new publication.

\section{Conclusion}

We have introduced a novel handheld diffuse optical breast scanning (DOB-Scan) probe for functional and compositional cross-sectional imaging of the breast tissue, which employs a multi-wavelength diffuse optical spectroscopy technique with four wavelengths encapsulated in an LED light source in the NIR spectrum range. The device provides four two-dimensional images of the breast tissue's function and relative composition. From the collected data at four different wavelengths, we are able to create cross-sectional optical absorption images for different chromophores in the breast tissue such as $\mathrm{Hb}$ and $\mathrm{HbO}_{2}$, which are indicators for cancerous tissue. A real-time data acquisition system was employed to add functional imaging advantages to the probe. The image reconstruction for each wavelength is performed at the rate of $24 \mathrm{~Hz}$ which is sufficient for functional imaging of biological activity. In this study, we evaluated performance of the probe by testing it on breast tissue-like phantoms. The test results show that the probe can create chromophores' relative composition images of the phantom. In addition, the results of the initial clinical study showed that the proposed method is able to provide accurate lesion information and has the potential to work as a complimentary to other types of imaging and diagnosis devices.

Although we obtained exciting results from clinical study, there are limitations to probe's performance. The maximum image depth, which is approximately half of the largest source-detector separation $(4.3 \mathrm{~cm})$, is limited by the size of the photodetector. If a lesion in the breast is located deeper than the maximum image depth, the probe is not able to detect it. We are currently searching for better solutions and improving the imaging algorithms to overcome the problem without sacrificing compactness and portability of the probe design.

This research has received ethical approval for clinical trials and currently the authors are testing the device on patients diagnosed with breast cancer. The promising imaging results of first two patients are presented in this paper. This study is ongoing and will accumulate a statistically viable number of case studies.

\section{Acknowledgments}

The Natural Sciences and Engineering Research Council of Canada (NSERC) provided partial financial support for this project. The authors thank Dr. Rhonda Janzen (with the Jim Pattison Outpatient Care and Surgery Center), Dr. Mojgan Jenab, M.Sc. student Zahra Haeiri (both from SFU) for conducting the clinical test, Sepideh M. Moqadam for her input in case design and M.Sc. student Mi Zhou at SFU to review and edit the paper.

\section{References}

1. American Cancer Society, Cancer Facts \& Figures (2016).

2. Canadian Cancer Society, Special Topic?: Predictions of the Future Burden of Cancer in Canada (2016).

3. L. Tabár and P. B. Dean, "A new era in the diagnosis and treatment of breast cancer," Breast J. 16(1), S2-S4 (2010).

4. H. D. Nelson, K. Tyne, A. Naik, C. Bougatsos, B. K. Chan, L. Humphrey, "Screening for breast cancer: Systematic evidence review update for the U. S. preventive services task force," Ann. Intern. Med. 151(10), 716-726 (2009).

5. A. Berrington de González, S. Darby, "Risk of cancer from diagnostic X-rays: Estimates for the UK and 14 other countries," Lancet 363(9406), 345-351 (2004).

6. C. M. Ronckers, C. A. Erdmann, C. E. Land, "Radiation and breast cancer: A review of current evidence," Breast Cancer Res. 7(1), 21-32 (2005).

7. A. Hassan and M. El-shenawee, "Review of electromagnetic techniques for breast cancer detection," IEEE Rev. Biomed. Eng. 4, 103-118 (2011).

8. J. Gonzalez, "Hand-held optical imager (Gen-2): Improved instrumentation and target detectability," J. Biomed. Opt. 17(8), 81402 (2012).

9. T. Vo-dinh, Biomedical Photonics Handbook, CRC Press (2003).

10. S. A. Prahl, "Optical properties spectra," http:// omlc.ogi.edu/spectra.

11. F. Bevilacqua, A. J. Berger, A. E. Cerussi, D. Jakubowski, B. J. Tromberg, "Broadband absorption spectroscopy in turbid media by combined frequency-domain and steady-state methods," Appl. Opt. 39(34), 6498-6507 (2000). 
12. P. Taroni, A. Pifferi, A. Torricelli, D. Comelli, R. Cubeddu, "In vivo absorption and scattering spectroscopy of biological tissues," Photochem. Photobiol. Sci. 2(2), 124-129 (2003).

13. D. A. Boas, C. Pitris, N. Ramanujam, Handbook of Biomedical Optics, CRC Press (2011).

14. T. J. Farrell, "A diffusion theory model of spatially resolved, steady-state diffuse reflectance for the noninvasive determination of tissue optical properties in vivo," Med. Phys. 19(4), 879 (1992).

15. S. L. Jacques, B. W. Pogue, "Tutorial on diffuse light transport," J. Biomed. Opt. 13(4), 41302 (2008).

16. M. L. Flexman, H. K. Kim, R. Stoll, M. A. Khalil, C. J. Fong, A. H. Hielscher, "A wireless handheld probe with spectrally constrained evolution strategies for diffuse optical imaging of tissue," Rev. Sci. Instrum. 83(3), 33108 (2012).

17. S. K. Biswas, K. Rajan, R. M. Vasu, "Diffuse optical tomographic imager using a single light source," J. Appl. Phys. 105(2), 24702 (2009).

18. S. J. Erickson, A. Godavarty, S. L. Martinez, J. Gonzalez, A. Romero, M. Roman, A. Nunez, J. Ge, S. Regalado, R. Kiszonas, C. Lopez-Penalver, "Hand-Held Optical Devices for Breast Cancer: Spectroscopy and 3-D Tomographic Imaging," IEEE J. Sel. Top. Quantum Electron. 18(4), 12981312 (2012).

19. S. J. Erickson and A. Godavarty, "Hand-held based near-infrared optical imaging devices: A review.," Med. Eng. Phys. 31(5), 495-509 (2009).

20. K. S. No, Q. Xie, R. Kwong, A. Cerussi, B. J. Tromberg, P. H. Chou, "HBS: A Handheld Breast Cancer detector based on frequency domain photon migration with full heterodyne," 2006 IEEE Biomed. Circuits Syst. Conf., London, pp. 114-117 (2006).

21. H. Yang, L. Xi, S. Samuelson, H. Xie, L. Yang, H. Jiang, "Handheld miniature probe integrating diffuse optical tomography with photoacoustic imaging through a MEMS scanning mirror," Biomed. Opt. Express 4(3), 427-432 (2013).

22. A. Cerussi, N. Shah, D. Hsiang, A. Durkin, J. Butler, B. J. Tromberg, "In vivo absorption, scattering, and physiologic properties of 58 malignant breast tumors determined by broadband diffuse optical spectroscopy," J. Biomed. Opt. 11(4), 44005 (2012).
23. M. Shokoufi, F. Golnaraghi, "Development of a handheld diffuse optical breast cancer assessment probe," J. Innov. Opt. Health Sci. 9(2), 1-10 (2015).

24. A. Godavarty, S. Rodriguez, Y.-J. Jung, S. Gonzalez, "Optical imaging for breast cancer prescreening," Breast Cancer Targets Ther. 2015(7), 193-209 (2015).

25. Q. Zhu, S. Tannenbaum, S. Kurtzman, "Optical tomography with ultrasound localization for breast cancer diagnosis and treatment monitoring," Surg. Oncol. Clin. N. Am. 16(2), 307-321 (2007).

26. T. J. Farrell, B. C. Wilson, M. S. Patterson, "The use of a neural network to determine tissue optical properties from spatially resolved diffuse reflectance measurements," Phys. Med. Biol. 37(12), 22812286 (1992).

27. V. G. Peters, D. R. Wyman, M. S. Patterson, G. L. Frank, "Optical properties of normal and diseased human breast tissues in the visible and near infrared," Phys. Med. Biol. 35(9), 1317-1334 (1990).

28. S. Fantini, A. Sassaroli, "Near-infrared optical mammography for breast cancer detection with intrinsic contrast," Ann. Biomed. Eng. 40(2), 398407 (2012).

29. R. Berg, S. Andersson-Engels, O. Jarlman, S. Svanbrg, "Time-gated viewing studies on tissue like phantoms," Appl. Opt. 35(19), 3432-3440 (1996).

30. S. T. Flock, S. L. Jacques, B. C. Wilson, W. M. Star, M. J. C. Van Gemert, "Optical properties of intralipid: A phantom medium for light propagation studies," Lasers Surg. Med. 12(5), 510-519 (1992).

31. S. B. Colak, M. B. van der Mark, G. W. t Hooft, J. H. Hoogenraad, E. S. van der Linden, F. a. Kuijpers, "Clinical optical tomography and NIR spectroscopy for breast cancer detection," IEEE J. Sel. Top. Quantum Electron. 5(4), 1143-1158 (1999).

32. H. J. van Staveren, C. J. Moes, J. van Marie, S. a Prahl, M. J. van Gemert, "Light scattering in Intralipid-10\% in the wavelength range of 400-1100 nm," Appl. Opt. 30(31), 4507-4514 (1991).

33. "Biomimic Optical Phantoms," http://www.ino.ca/ en/products/.

34. M. Shokoufi, "Multi - modality breast cancer assessment tools using diffuse optical and electrical impedance spectroscopy," Simon Fraser University (2016). 